\title{
Regulatory authorities' preparedness to avert poor quality alcohol based hand sanitizers during COVID-19 pandemic in Ethiopia:A commentary
}

\begin{abstract}
The World Health Organization recommends the use of ethyl alcohol or isopropyl alcoholbased hand sanitizers to control the spread of the COVID 19 pandemic. As a result, a number of temporary guidelines and policies are being claimed among different countries of the world by waiving restrictions on the production of hand sanitizers which allow manufacturers, including alcohol beverage manufacturers, to produce alcohol based hand sanitizers in midst of COVID-19 pandemic. Under such circumstances there will be a high chance of producing substandard and counterfeit alcohol based sanitizers by unscrupulous firms. In addition, the fragile regulatory authority system coupled with risk of using poor quality sanitizers highly requires the active preparedness of regulatory authorities in resource limited setting, including Ethiopia. Therefore, the current commentary highly recommends the regulatory authority of Ethiopia to actively counterpart the spread of poor quality sanitizers. Moreover, assessment of the quality of alcohol based hand sanitizer circulating in Ethiopia, low income country should be undertaken as soon as possible to assure its quality.
\end{abstract}

Volume 8 Issue 6 - 2020

\author{
Gemmechu Hasen, ${ }^{1,2}$ Sultan Suleman ${ }^{1,2}$ \\ 'School of Pharmacy, Faculty of Health Sciences, Jimma \\ University, Ethiopia \\ ${ }_{2}^{2}$ Jimma University Laboratory of Drug Quality (JuLaDQ), Jimma \\ University, Ethiopia
}

Correspondence: Gemmechu Hasen, School of Pharmacy, Faculty of Health Sciences, P.O. Box: 378, Jimma University, Jimma, Ethiopia, Tel +251916973323,

Email gemmechuhasen2009@gmail.com

Received: October 19, 2020 | Published: November 12, 2020
In early December 2019, an outbreak of coronavirus disease 2019 (COVID-19), caused by a novel severe acute respiratory syndrome coronavirus 2 (SARS-CoV-2), emerged in Wuhan City, Hubei Province, China. ${ }^{1}$ On January 30, 2020 the World Health Organization (WHO) declared the outbreak as a Public Health Emergency of International Concern. ${ }^{2}$ Since then, the WHO has identified formulations for the local preparation of alcohol-based hand rub formulations as gold standard, and the key tools being utilized to control the spread of the novel SARS-CoV-2 pandemic. ${ }^{3}$ In line with these, various governments around the world have promoted the use of ethyl alcohol or isopropyl alcohol-based hand rubs for hand hygiene. Moreover, a number of temporary guidelines and policies are being proclaimed among different countries of the world by waiving restrictions on the production of hand sanitizers which allow manufacturers, including alcohol beverage manufacturers, to produce alcohol based hand sanitizers in midst of COVID-19 pandemic. ${ }^{4}$ Thus under such circumstances, there will be a high chance of producing substandard and counterfeit alcohol based sanitizers by unscrupulous firms.

For instance, the FDA Punjab of India collected a total of 75 samples of alcohol-based sanitizers, of which 25 samples had been tested, and 17 were found to be of sub-standard quality while five sample were misbranded, giving wrong information about the product. ${ }^{5}$ Given the popularity of hand sanitizers and their need to prevent the spread of COVID-19, counterfeit alcohol-based hygiene poses a public health risk. Accordingly, in the context of this outbreak of COVID-19, researchers have been strongly recommending the regulatory and public health bodies to take an active role in ensuring the safety and quality of antimicrobial products such as alcohol-based hand sanitizers at every stage of the products' lifecycle, including distribution, manufacture and import. ${ }^{6}$ However, the public health impact of the pandemic, and weak regulatory system with less implementation of existing regulatory system in low income countries is of grave concern as they are major concentrated on control COVID-19 pandemic using alcohol based hand sanitizer produced without assurance of its quality.

This situation is very severe in Ethiopia in which unknown firms are frequently producing hand sanitizers. Moreover, no clear guideline is prepared by EFDA despite different firms are producing huge amounts of hand sanitizers. As stated by Ethiopia's Ministry of Trade and Industry said on Thursday March 30, 2020 close to 40 companies have begun to produce sanitizer in a bid to curb the corona virus pandemic. The government has also distributed over 2.5milion litters of technical alcohol collected from Sugar factories to be used as input sanitizer production. ${ }^{7}$ Moreover, private pharmacies and different university in Ethiopia are producing this sanitizer without any sat restrictions by authority. This may bring a chance for unscrupulous manufactures to spread substandard and counterfeit hand sanitizer in Ethiopia. This in turn may lead to exacerbate already limited prevention towards spread of COVID-19. The discussion above identifies the regulatory authorities' preparedness to avoid poor quality alcohol based hand sanitizer as a serious public health risk, especially in the context of the outbreak of COVID-19.

The fragile regulatory authority system coupled with risk of using poor quality sanitizers highly requires the active preparedness of regulatory authorities in resource limited setting. It is recommended the regulatory authority of low income countries should be actively prepared to counterpart the spread of poor quality sanitizers while controlling of COVID-19 pandemic too. Moreover, assessment of the quality of alcohol based hand sanitizer circulating in Ethiopia, low income countries should be undertaken as soon as possible to assure its quality.

\section{Ethical approval}

Not applicable. 


\section{Source of funding}

The author(s) received no financial support for the research, authorship, and/or publication of this article.

\section{Author contribution}

Gemmechu H drafted the commentary. Gemmechu H and Sultan S provided critical input and edits. Both authors read and approved the final manuscript.

\section{Registration of research studies}

a. Name of the registry: Not applicable

b. Unique Identifying number or registration ID: Not applicable

c. Hyperlink to your specific registration (must be publicly accessible and will be checked): Not applicable

\section{Guarantor}

Gemmechu Hasen.

\section{Consent}

Not applicable.

\section{Availability of data and materials}

Not applicable.

\section{Provenance and peer review}

Not commissioned, externally peer reviewed.

\section{Acknowledgments}

Not applicable.

\section{Declaration of competing interest}

No competing interests exist.

\section{References}

1. Harapan H, Itoh N, Yufika A, et al. Coronavirus disease 2019 (COVID-19): A literature review. J Infect Public Health. 2020;13(5):667-673.

2. WHO. COVID 19 Public Health Emergency of International Concern (PHEIC). Global research and innovation forum: towards a research roadmap; 2020.

3. Nyamweya N, Abuga K. COVID-19: Quality and safety of hand sanitizers considerations. University of Nairobi; 2020.

4. U.S. Department of Health and Human Services. Temporary Policy for Preparation of Certain Alcohol-Based Hand Sanitizer Products during the Public Health Emergency (COVID-19) Guidance for Industry; 2020.

5. Kumar V. Substandard hand sanitizers sold in Punjab: Food and drug administration report; 2020.

6. Jairoun AA, Sabaa SA-H, Shahwan M. The pandemic of COVID-19 and its implications for the purity and authenticity of alcohol-based hand sanitizers: The health risks associated with falsified sanitizers and recommendations for regulatory and public health bodies. Res Soc Adm Pharm. 2020;S1551-7411(20):30393-30394.

7. Sahlu S. Ethiopian Monitor. Close to 40 Firms Start Sanitizer Production; 2020 . 